\title{
Proposal of the performance status combined Japan Integrated Staging system in hepatocellular carcinoma complicated with cirrhosis
}

\author{
HIROKI NISHIKAWA, RYUICHI KITA, TORU KIMURA, MASATSUGU ENDO, \\ YOSHIAKI OHARA, AZUSA SAKAMOTO, SUMIO SAITO, NORIHIRO NISHIJIMA, \\ AKIHIRO NASU, HIDEYUKI KOMEKADO and YUKIO OSAKI
}

Department of Gastroenterology and Hepatology, Osaka Red Cross Hospital, Osaka 543-0027, Japan

Received February 13, 2015; Accepted March 30, 2015

DOI: 10.3892/ijo.2015.2969

\begin{abstract}
The present study examined the prognostic ability of our proposed performance status combined Japan Integrated Staging (PS-JIS) system in hepatocellular carcinoma (HCC) patients with liver cirrhosis (LC) comparing with other four prognostic systems including original JIS system, the Barcelona Clinic Liver Cancer classification system, TNM classification system and the Cancer of the Liver Italian Program (CLIP) scoring system. A total of 1,170 HCC patients complicated with LC were analysed. The disease was staged for all analysed patients by means of the five staging systems. The cumulative overall survival (OS) rate was calculated by Kaplan-Meier method and tested by log-rank test. We also examined prognostic factors associated with OS using univariate and multivariate analyses and compared the prognostic ability in each prognostic system using concordance index (c-index) at 1-, 3- and 5-year time-points. Overall significance in each prognostic system was $P<0.001$. In the multivariate analyses, tumor number, Child-Pugh classification, PS, initial treatment modality and several laboratory parameters were significant independent predictors linked to OS. For all cases, in each time-point, the c-index of PS-JIS system was the highest among five staging systems $(0.847$, 0.816 and 0.808 , respectively), indicating that PS-JIS system has the best predictability among these staging systems. According to subgroup analyses stratified by initial treatment modality, in patients treated with surgical resection $(n=205)$, CLIP scoring system had the highest c-index at every timepoint, whereas in patients treated with percutaneous ablative therapies $(n=632)$ at 3 - and 5-year time-point and in those with
\end{abstract}

Correspondence to: Dr Hiroki Nishikawa, Department of Gastroenterology and Hepatology, Osaka Red Cross Hospital, 5-30 Fudegasaki-cho, Tennoji-ku, Osaka 543-0027, Japan

E-mail: h-nishikawa@osaka-med.jrc.or.jp

Key words: hepatocellular carcinoma, liver cirrhosis, performance status, Japan Integrated Staging, predictability transcatheter arterial therapies $(n=281)$ at every time-point, the c-index of PS-JIS system was the highest. In conclusion, the proposed PS-JIS score can be a useful prognostic system for HCC patients complicated with liver cirrhosis.

\section{Introduction}

Clinical staging for malignancies provides a useful guidance for predicting survival and for deciding optimal treatment strategies (1). Design of a cancer staging system depends on the identification of individual prognostic factors that can predict survival of cancer patients (1-3). Unlike other solid tumors, the prognosis and treatment strategies for subjects with hepatocellular carcinoma (HCC) depend not only on the tumor characteristics but also on the degree of liver function (2-9). Based on the identification of relevant predictors for both the tumor burden and liver functional reserve, several staging systems for HCC including both aspects had been proposed.

In 1998, the Cancer of the Liver Italian Program (CLIP) proposed a new scoring system (CLIP scoring system) that accounts for both tumor characteristics and liver function relevant to prognostic evaluation for HCC patients. This score consisted of four variables of Child-Pugh classification, $\alpha$-fetoprotein (AFP) value, tumor morphology and portal vein invasion and its prognostic ability has been validated in several countries (2-5). On the other hand, Llovet et al (6) proposed Barcelona Clinic Liver Cancer (BCLC) classification system for HCC consisting of tumor characteristics, associated liver disease and the Eastern Cooperative Oncology Group (ECOG) performance status (ECOG-PS) in 1999. This is the only system that provides treatment recommendations for each $\mathrm{HCC}$ stage based on the best treatment strategies currently available and has been externally validated in the United States and Europe and endorsed by both the European Association for the Study of the Liver (EASL) and the American Association for the Study of Liver Diseases (AASLD). (7-9) In Japan, in 2003, Kudo et al proposed the Japan Integrated Staging (JIS) system consisting of Child-Pugh classification and HCC stage as defined by TNM classification by the Liver Cancer Study Group of Japan (LCSGJ) as a prognostic system and they demonstrated that this system was a better prognostic system 
Table I. Definition of the proposed performance status combined Japan Integrated Staging system.

\begin{tabular}{lcccr}
\hline & \multicolumn{4}{c}{ Score } \\
\cline { 2 - 5 } Variables & 0 & 1 & 2 & 3 \\
\hline Child-Pugh stage & A & B & C & \\
TNM stage (LCSGJ) & I & II & III & IV \\
Performance status & 0 & 1 & $>2$ & \\
\hline
\end{tabular}

LCSGJ; Liver Cancer Study Group of Japan.

than CLIP scoring system using a large cohort $(n=4525)$ (10-12). Currently, more than ten staging classification for HCC are available (13).

The major difference between CLIP scoring system, BCLC classification system and JIS system is that only BCLC classification system included ECOG-PS as a variable. The PS scale is a major survival determinant in patients with $\operatorname{HCC}(14,15)$. Especially in HCC patients complicated with liver cirrhosis (LC), those with deteriorated PS are encountered in the daily clinical practice. This is probably due to the fact that LC related complications such as ascites, encephalopathy and muscle wasting lead to deterioration of PS (16) Furthermore, in Japan, the proportion of aged HCC patients with potentially poorer PS has been increasing (17).

Currently, there are two modified JIS system: biomarker combined JIS system and the model for end stage liver disease-based JIS system $(18,19)$. In the present study, on the basis of above, we herein propose a PS combined JIS (PS-JIS) system for HCC patients with LC. The aims of the present study were to examine the prognostic ability of our proposed PS-JIS system in HCC patients with LC comparing with other prognostic systems.

\section{Patients and methods}

Patients. A total of 1,170 consecutive treatment-naïve patients diagnosed with HCC complicated with LC were admitted to the Department of Gastroenterology and Hepatology, Osaka Red Cross Hospital, Japan, between March 2004 and June 2014. LC was determined based on radiologic findings including typical computed tomography (CT) or ultrasound findings, laboratory parameters and/or histological findings obtained by surgical specimens or liver biopsy. PS was evaluated by using the ECOG performance scale ranging from 0 (asymptomatic) to 4 (confined to bed).

As reported by Kudo et al JIS score was calculated by summation of TNM stage score by the LCSGJ (stage I, 0; stage I, 1; stage III, 2; and stage IV, 3) and Child-Pugh classification (A, 0; B, 1; and C, 2) $(10,11)$. Our proposed PS-JIS system was calculated by summation of TNM stage score by the LCSGJ (stage I, 0; stage II, 1; stage III, 2; and stage IV, 3), Child-Pugh classification (A, 0; B, 1; and C,2) and PS (PS 0, 0 ; PS 1, 1; and PS >2, 2). Thus, scores of our proposed PS-JIS system ranged from 0 to 7 (Table I). The disease was staged for all analysed patients by means of five staging systems including JIS system, our proposed PS-JIS system, BCLC classification system, TNM classification system and CLIP scoring system. We examined the prognostic ability in each prognostic system using concordance index (c-index) as described later. Furthermore, we examined prognostic factors associated with overall survival (OS) using univariate and multivariate analyses. The following data were used for the current analyses: gender, age, tumor number, maximum tumor size, Child-Pugh classification, ECOG-PS, initial treatment modality, cause of liver disease, aspartate aminotransferase (AST), alanine aminotransferase (ALT), alkaline phosphatase (ALP), gamma glutamyl transpeptidase (GGT), platelet count and tumor markers.

Prior to therapy for $\mathrm{HCC}$, written informed consent for HCC therapy was obtained from all subjects. The ethics committee of our department approved the protocol for this study. The present study comprised a retrospective analysis of patients' medical records in our database and all treatments were performed in an open-label manner.

Diagnosis of HCC and HCC therapy. HCC was diagnosed based on the results from abdominal ultrasound and dynamic CT scan (hyper-attenuation during the arterial phase in the entire or part of the tumor, and hypo-attenuation in the portal-venous phase) and/or magnetic resonance imaging (MRI) mainly as recommended by the AASLD (14). Arterial and portal phase dynamic CT images were obtained $~ 30$ and $120 \mathrm{sec}$ after injection of contrast material. In our hospital, abdominal angiography combined with CT (angio-CT) was routinely performed before therapy for HCC after obtaining informed consent for performing abdominal angiography. This was performed based on the fact that this technique was useful for detecting small satellite nodules as reported by Yamasaki et al (20). Then, we confirmed HCC using CT during hepatic arteriography (CTHA) and CT during arterialportography (CTAP). Vascular invasion was determined by dynamic CT and/or angio-CT. During initial evaluation for HCC, a chest X-ray was performed, and if abnormal, a chest CT scan was done. Bone scintigraphy or brain CT scan or MRI was done if there was any suggesting symptoms or clinical indication. As for HCC therapy, the most appropriate treatment modality for each HCC patient was selected through discussion with surgeons, hepatologists and radiologists $(21,22)$. Best supportive care was provided when treatment efficacy was considered limited or patients refused therapy for HCC. In the present analysis, there was no patient treated with liver transplantation.

Follow-up after initial therapy for HCC. Follow-up observation consisted of regularly blood tests and monitoring of tumor markers, including AFP and des- $\gamma$-carboxy prothrombin (DCP), which was measured using a chemiluminescent enzyme immunoassay (Lumipulse PIVKAII Eisai; Eisai Co., Ltd., Tokyo, Japan). Dynamic CT scan was performed every 3-4 months after initial therapy for HCC. When HCC recurrence or disease progression was detected based on radiologic findings, most appropriate therapy was performed in each patient.

Statistical analysis. In the present study, OS was the only end point. Data were analyzed using univariate and multivariate 
analyses. To analyze the significance of prognostic predictors, continuous variables were divided by the median values for all cases $(n=1,170)$ and treated as dichotomous covariates. The cumulative OS rate was calculated by Kaplan-Meier method and tested by log-rank test. A Cox proportional hazard model via a stepwise forward method was used for multivariate analyses of factors with P-value $<0.05$ in univariate analyses. These statistical methods were used to estimate the interval from the date of diagnosis for HCC until the date of death or last follow-up date.

To evaluate the discriminatory ability for predicting survival, we assessed the accuracy of prediction of death at 1, 3 and 5 years for each scoring system. This score was assessed by calculating the area under the receiver operating characteristic (ROC) curve for each score [which is equivalent to the concordance index (c-index)] (23). To perform this test, subjects censored before 1, 3 and 5 years were excluded from the analysis. $\mathrm{C}$-index of 0.5 indicates that the model is no better than chance at making a prediction of membership in a group and a value of 1.0 indicates that the model perfectly identifies those within a group and those not. Models are typically considered reasonable when the c-index is $>0.70$ (24).

Data were analyzed using SPSS software (SPSS, Inc., Chicago, IL, USA) for Microsoft Windows. Data are expressed as median value (range). A P-value $<0.05$ were considered to be statistically significant.

\section{Results}

Patient demographic characteristics. Baseline demographic characteristics of analysed patients $(n=1,170)$ are shown in Table II. They included 742 males and 446 female. The median age was 70 (range, 32-91). There were 804 patients in Child-Pugh A, 303 in Child-Pugh B and 63 in Child-Pugh C. In terms of ECOG-PS, they included 885 subjects in PS 0, 148 in PS 1, 93 in PS 2, 29 in PS 3 and 15 in PS 4, respectively. The median maximum tumor diameter was $2.5 \mathrm{~cm}$ (range, $0.5-18 \mathrm{~cm}$ ). The proportion of hepatitis virus-related (hepatitis B, C or B and C) was $81.6 \%$ (955/1170). In the present analyses, AFP values were missing from two subjects and DCP values were missing from 15 subjects.

Initial treatment for HCC, overall survival and causes of death for all cases. As an initial therapy for HCC, surgical resection (SR) was performed in 205 patients, percutaneous ablative therapies (PATs) such as radiofrequency ablation (RFA) or percutaneous ethanol injection in 632, trancatheter arterial chemotherapy with or without embolization (transcatheter arterial therapies, TATs) in 281, molecular targeted therapy such as sorafenib in four, radiation therapy in two and no specific therapy in 13 .

The median follow-up period was 2.8 years. The 1-, 3 and 5-year cumulative OS rates were $86.3,62.3$ and $43.5 \%$, respectively (Fig. 1). During follow-up period, there were 625 $(53.4 \%)$ deaths. The causes of death were HCC recurrence in 346 patients, liver failure in 204 patients and miscellaneous causes in 75 patients, respectively.

Univariate and multivariate analyses of factors contributing to $O S$. Using univariate analyses of factors contributing to
Table II. Baseline characteristics $(n=1,170)$.

\begin{tabular}{lc}
\hline Variables & $\begin{array}{c}\text { No. or median value } \\
\text { (range) }\end{array}$ \\
\hline Age (years) & $70(32-91)$ \\
Gender, male/female & $724 / 446$ \\
Causes of liver disease, & \\
B/C/non-B non-C/B and C & $120 / 816 / 215 / 19$ \\
Child-Pugh, A/B/C & $804 / 303 / 63$ \\
ECOG performance status, & \\
0/1/2/3/4 & $885 / 148 / 93 / 29 / 15$ \\
Maximum tumor size (cm) & $2.5(0.5-18)$ \\
Tumor number, single/multiple & $632 / 538$ \\
AST (IU/l) & $57(9-536)$ \\
ALT (IU/l) & $44(3-438)$ \\
Total bilirubin (mg/dl) & $0.9(0.2-19.6)$ \\
Serum albumin (g/dl) & $3.7(1.1-5.1)$ \\
ALP (IU/l) & $348(87-3344)$ \\
GGT (IU/l) & $64(10-1460)$ \\
Prothrombin time $(\%)$ & $80(32-145)$ \\
Platelets $\left(x 10^{4} / m^{3}\right)$ & $9.2(1.6-37.3)$ \\
AFP (ng/ml ${ }^{\mathrm{a}}$ & $29.2(1.4-843700)$ \\
DCP (mAU/ml) & $55(1-328340)$ \\
&
\end{tabular}

ECOG, the Eastern Cooperative Oncology Group; AST, aspartate aminotransferase; ALT, alanine aminotransferase; ALP, alkaline phosphatase; GGT, gamma glutamyl transpeptidase; AFP, $\alpha$-fetoprotein; DCP, des- $\gamma$-carboxy prothrombin; ${ }^{a}$ missing data, $n=2$; ${ }^{b}$ missing data, $\mathrm{n}=15$.

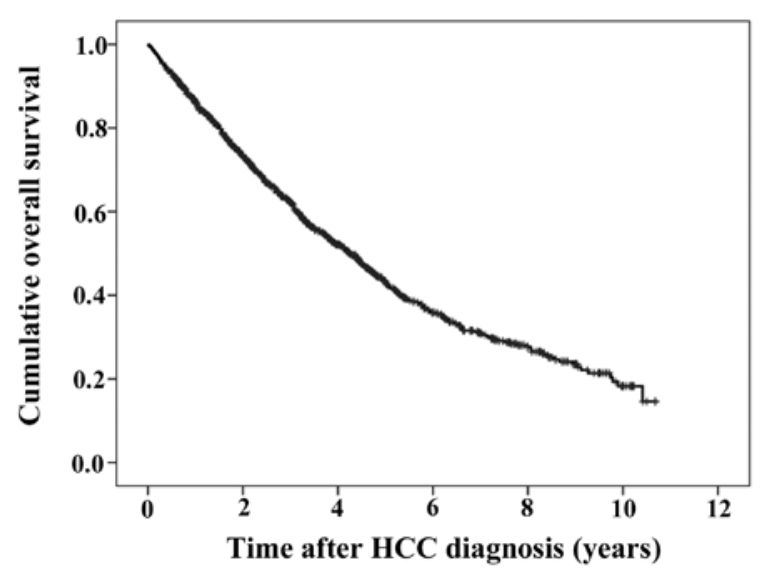

Figure 1. Cumulative overall survival for all cases $(n=1,170)$. The $1-, 3$ - and 5-year cumulative overall survival rates were $86.3,62.3$ and $43.5 \%$, respectively.

OS, tumor number $(\mathrm{P}<0.001)$, maximum tumor size $>2.5 \mathrm{~cm}$ $(\mathrm{P}<0.001)$, Child-Pugh classification $(\mathrm{P}<0.001)$, PS $(\mathrm{P}<0.001)$, initial treatment modality $(\mathrm{P}<0.001)$, AST $>57$ IU/1 $(\mathrm{P}<0.001)$, ALP >348 IU/1 (P<0.001), GGT >64 IU/1 $(\mathrm{P}<0.001)$, AFP $>29.2 \mathrm{ng} / \mathrm{ml}(\mathrm{P}<0.001)$ and $\mathrm{DCP}>55 \mathrm{mAU} / \mathrm{ml}(\mathrm{P}<0.001)$ were found to be significant factors associated with OS (Table III). 
Table III. Univariate and multivariate analyses of factors contributing to overall survival $(\mathrm{n}=1,170)$.

\begin{tabular}{|c|c|c|c|c|}
\hline \multirow[b]{2}{*}{ Variables } & \multirow[b]{2}{*}{$\mathrm{n}$} & \multirow[b]{2}{*}{ Univariate analysis } & \multicolumn{2}{|c|}{ Multivariate analysis } \\
\hline & & & $\begin{array}{l}\text { Hazard ratio } \\
(95 \% \mathrm{CI})\end{array}$ & P-value ${ }^{a}$ \\
\hline Gender, male vs. female & $724 / 446$ & 0.081 & & \\
\hline Age (years), $>70$ vs. $<70$ & $615 / 555$ & 0.175 & & \\
\hline Tumor number, single vs. multiple & $632 / 538$ & $<0.001$ & $0.587(0.493-0.698)$ & $<0.001$ \\
\hline Maximum tumor size $(\mathrm{cm}),>2.5$ vs. $<2.5$ & $599 / 571$ & $<0.001$ & & \\
\hline Child-Pugh, A vs. B vs. C & $804 / 303 / 63$ & $<0.001$ & & \\
\hline Child-Pugh A & & & 1.000 (reference) & \\
\hline Child-Pugh B & & & $0.537(0.380-0.759)$ & $<0.001$ \\
\hline Child-Pugh C & & & $0.300(0.212-0.425)$ & $<0.001$ \\
\hline ECOG-PS, 0 vs. 1 vs. $>2$ & $885 / 148 / 137$ & $<0.001$ & & \\
\hline PS 0 & & & 1.000 (reference) & \\
\hline PS 1 & & & $0.731(0.539-0.992)$ & 0.044 \\
\hline $\mathrm{PS}>2$ & & & $0.437(0.339-0.563)$ & $<0.001$ \\
\hline $\begin{array}{l}\text { Initial treatment modality, } \\
\text { SR/PATs/others/none }\end{array}$ & $205 / 632 / 287 / 46$ & $<0.001$ & & \\
\hline $\mathrm{SR}$ & & & 1.000 (reference) & \\
\hline PATs & & & $0.742(0.479-1.147)$ & 0.179 \\
\hline Others & & & $0.468(0.304-0.723)$ & 0.001 \\
\hline None & & & $0.392(0.243-0.631)$ & $<0.001$ \\
\hline Cause of liver disease, virus related vs. NBNC & $955 / 215$ & 0.511 & & \\
\hline AST (IU/l),$>57$ vs. $<57$ & $593 / 577$ & $<0.001$ & $0.710(0.601-0.839)$ & $<0.001$ \\
\hline $\operatorname{ALT}(\mathrm{IU} / \mathrm{l}),>44$ vs. $<44$ & $587 / 583$ & 0.132 & & \\
\hline $\operatorname{ALP}(\mathrm{IU} / \mathrm{l}),>348$ vs. $<348$ & $586 / 584$ & $<0.001$ & $0.726(0.614-0.858)$ & $<0.001$ \\
\hline GGT (IU/l), $>64$ vs. $<64$ & $590 / 580$ & $<0.001$ & & \\
\hline Platelet count $\left(\times 10^{4} / \mathrm{mm}^{3}\right),>9.2$ vs. $<9.2$ & $590 / 580$ & 0.783 & & \\
\hline $\operatorname{AFP}(\mathrm{ng} / \mathrm{ml}),>29.2$ vs. $<29.2^{\mathrm{b}}$ & $584 / 584$ & $<0.001$ & $0.832(0.704-0.983)$ & 0.030 \\
\hline $\mathrm{DCP}(\mathrm{mAU} / \mathrm{ml}),>55 \mathrm{vs} .<55^{\mathrm{c}}$ & $580 / 575$ & $<0.001$ & $0.465(0.391-0.553)$ & $<0.001$ \\
\hline
\end{tabular}

CI, confidence interval; ECOG-PS, the Eastern Cooperative Oncology Group performance status; SR, surgical resection; PATs, percutaneous ablative therapies; NBNC, non-B and non-C; AST, aspartate aminotransferase; ALT, alanine aminotransferase; ALP, alkaline phosphatase; GGT, gamma glutamyl transpeptidase; AFP, $\alpha$-fetoprotein; DCP, des- $\gamma$-carboxy prothrombin; ${ }^{\mathrm{a} C o x}$ proportional hazard model; ${ }^{\mathrm{b}}$ missing data, $\mathrm{n}=2$; ${ }^{\mathrm{c}}$ missing data, $\mathrm{n}=15$.

The multivariate analyses involving ten factors with $\mathrm{P}<0.05$ in the univariate analysis demonstrated that tumor number, Child-Pugh classification ( $\mathrm{P}<0.001$ for $\mathrm{B}$ and $\mathrm{P}<0.001$ for $\mathrm{C}$ as reference of $\mathrm{A}$ ), $\mathrm{PS}(\mathrm{P}=0.044$ for $\mathrm{PS} 1$ and $\mathrm{P}<0.001$ for $\mathrm{PS}>2$ as reference of PS 0$)$, initial treatment modality $(\mathrm{P}=0.001$ for other treatments than SR or PATs and $\mathrm{P}<0.001$ for no specific therapy as reference of SR), AST $>57$ IU/1 $(\mathrm{P}<0.001)$, ALP $>348$ IU/l $(\mathrm{P}<0.001)$, AFP $>29.2 \mathrm{ng} / \mathrm{ml}(\mathrm{P}=0.003)$ and DCP $>55 \mathrm{mAU} / \mathrm{ml}(\mathrm{P}<0.001)$ were significant independent predictors linked to OS. The hazard ratios (HRs), 95\% confidence intervals (CIs) and P-values for these factors are detailed in Table III.

Comparison of PS-JIS score and existing criteria for HCC for all cases using c-index. Kaplan-Meier curves of OS according to five criteria are demonstrated: JIS system, PS-JIS system, BCLC classification system, TNM classification system and CLIP scoring system (Figs. 2-6). Number and median OS of patients with each score are demonstrated in Table IV. P-values between adjacent groups in each system are shown in Table IV. Overall significance in each prognostic system was $\mathrm{P}<0.001$. All P-values between adjacent groups in each system reached significance except for differences in PS-JIS score 4 and 5 $(\mathrm{P}=0.873)$, PS-JIS score 6 and $7(\mathrm{P}=0.199)$ and CLIP score 4 and 5 or $6(\mathrm{P}=0.082)$.

To examine predictability of each staging system, we compared them using the c-index. The 1-year c-indexes of JIS system, PS-JIS system, BCLC classification system, TNM classification system and CLIP scoring system were 0.841, 0.847, $0.815,0.819$ and 0.817 , respectively. The 3 -year c-indexes of JIS 
Table IV. Patient survival according to different staging system.

\begin{tabular}{|c|c|c|c|c|}
\hline Staging system & MST (years) & $95 \% \mathrm{CI}$ & P-value (overall) & P-value in each adjacent group \\
\hline JIS system & & & $<0.001$ & \\
\hline $0(\mathrm{n}=222)$ & 6.64 & $4.87-8.41$ & & 0 vs. $1,0.001$ \\
\hline $1(\mathrm{n}=408)$ & 5.72 & $4.62-6.82$ & & 1 vs. $2,<0.001$ \\
\hline $2(\mathrm{n}=297)$ & 3.15 & $2.57-3.73$ & & 2 vs. $3,<0.001$ \\
\hline $3(\mathrm{n}=139)$ & 1.71 & $1.48-1.94$ & & 3 vs. $4,<0.001$ \\
\hline $4(n=86)$ & 0.75 & $0.63-0.87$ & & 4 vs. $5,0.001$ \\
\hline $5(n=18)$ & 0.23 & $0.17-0.29$ & & \\
\hline PS-JIS system & & & $<0.001$ & \\
\hline $0(\mathrm{n}=187)$ & 8.31 & $5.90-10.72$ & & 0 vs. $1,0.015$ \\
\hline $1(\mathrm{n}=348)$ & 6.64 & $5.60-7.68$ & & 1 vs. $2,<0.001$ \\
\hline $2(\mathrm{n}=288)$ & 3.59 & $2.96-4.22$ & & 2 vs. $3,<0.001$ \\
\hline $3(n=170)$ & 2.38 & $1.94-2.82$ & & 3 vs. $4,<0.001$ \\
\hline $4(n=98)$ & 1.41 & $0.99-1.83$ & & 4 vs. $5,0.873$ \\
\hline $5(n=35)$ & 1.54 & $0.87-2.21$ & & 5 vs. $6,0.003$ \\
\hline $6(n=34)$ & 0.65 & $0.02-1.28$ & & 6 vs. $7,0.199$ \\
\hline $7(\mathrm{n}=10)$ & 0.21 & $0.01-0.48$ & & \\
\hline BCLC classification system & & & $<0.001$ & \\
\hline 0 (very early stage, $n=187$ ) & 8.31 & $5.90-10.72$ & & 0 vs. $\mathrm{A},<0.001$ \\
\hline A (early stage, $n=427$ ) & 5.47 & $4.61-6.33$ & & A vs. B, $<0.001$ \\
\hline B (intermediate stage, $n=194$ ) & 3.07 & $2.61-3.53$ & & B vs. $\mathrm{C},<0.001$ \\
\hline $\mathrm{C}($ advanced stage, $\mathrm{n}=265)$ & 2.10 & $1.48-2.72$ & & C vs. D, $<0.001$ \\
\hline $\mathrm{D}$ (end stage, $\mathrm{n}=97$ ) & 1.07 & $0.66-1.49$ & & \\
\hline TNM classification system & & & $<0.001$ & \\
\hline Stage I $(n=290)$ & 6.32 & $5.68-6.96$ & & I vs. II, 0.001 \\
\hline Stage II $(n=463)$ & 4.93 & $4.26-5.60$ & & II vs. III, $<0.001$ \\
\hline Stage III $(n=290)$ & 2.71 & $2.28-3.14$ & & III vs. IV, $<0.001$ \\
\hline Stage IV $(n=127)$ & 0.66 & $0.49-0.83$ & & \\
\hline CLIP scoring system ${ }^{\mathrm{a}}$ & & & $<0.001$ & \\
\hline $0(\mathrm{n}=415)$ & 7.36 & $6.25-8.47$ & & 0 vs. $1,<0.001$ \\
\hline $1(n=422)$ & 4.15 & $3.69-4.61$ & & 1 vs. $2,<0.001$ \\
\hline $2(\mathrm{n}=192)$ & 2.51 & $2.04-2.98$ & & 2 vs. $3,<0.001$ \\
\hline $3(n=87)$ & 1.02 & $0.72-1.32$ & & 3 vs. $4,0.001$ \\
\hline $4(n=34)$ & 0.37 & $0.20-0.54$ & & 4 vs. 5 or $6,0.082$ \\
\hline 5 or $6(n=18)$ & 0.26 & $0.20-0.32$ & & \\
\hline
\end{tabular}

JIS, Japan Integrated Staging; PS-JIS, performance status combined Japan Integrated Staging; BCLC, Barcelona Clinic Liver Cancer; CLIP,

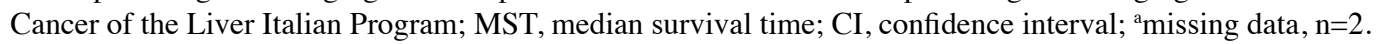

system, PS-JIS sytem, BCLC classification system, TNM classification system and CLIP scoring system were $0.797,0.816$, $0.778,0.754$ and 0.777 , respectively. The 5-year c-indexes of JIS system, PS-JIS system, BCLC classification system, TNM classification system and CLIP scoring system were 0.775 , $0.808,0.775,0.723$ and 0.776 , respectively. Collectively, in each time-point, the c-index of PS-JIS score was the highest in these staging systems, indicating that PS-JIS score had the best predictability among these staging systems (Table V).
Comparison of PS-JIS system and existing criteria for HCC according to initial treatment modality. We also performed subgroup analyses according to initial treatment modality using c-index. In patients treated with SR $(n=205)$, in 1-, 3and 5-year c-index, CLIP scoring system had the highest value among five staging systems (c-index, 0.739, 0.722 and 0.681 , respectively). In patients treated with PATs $(n=632)$, in 1-year c-index, BCLC classification system had the highest value (c-index, 0.740), whereas in 3- and 5-year c-index, PS-JIS 


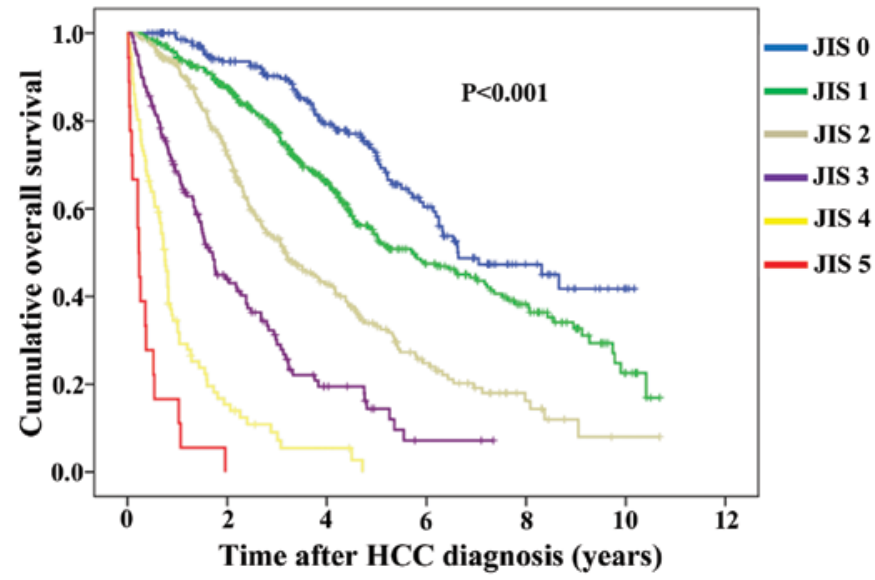

Figure 2. Kaplan-Meier survival curves for HCC patients by Japan Integrated Staging (JIS) system (overall significance, $\mathrm{P}<0.001$ ).

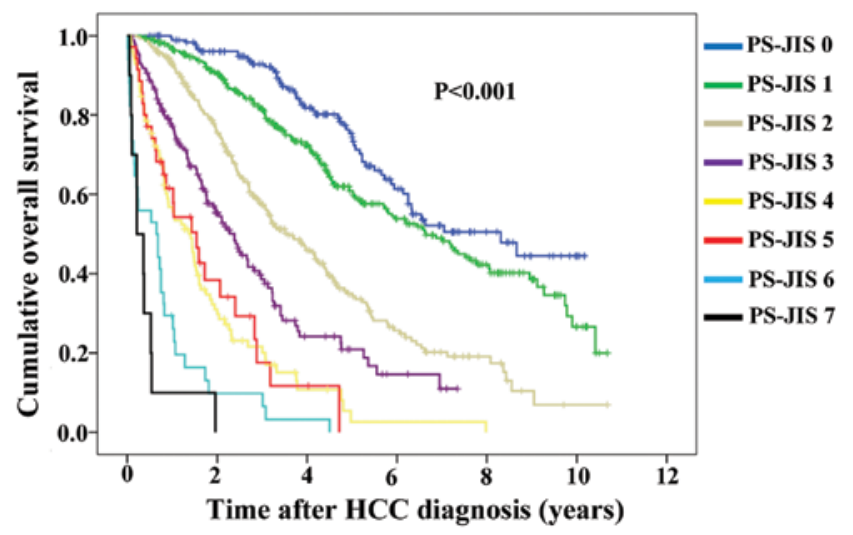

Figure 3. Kaplan-Meier survival curves for HCC patients by the proposed performance status combined Japan Integrated Staging (PS-JIS) system (overall significance, $\mathrm{P}<0.001$ ).

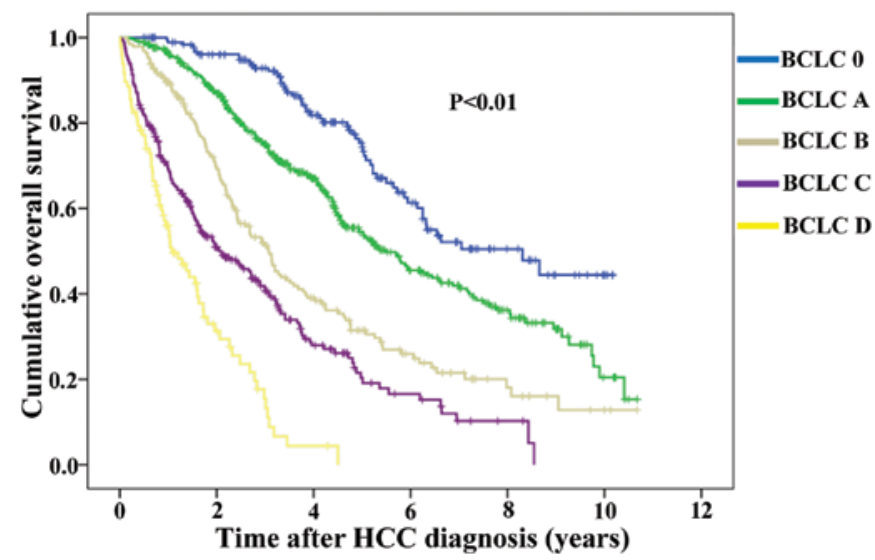

Figure 4. Kaplan-Meier survival curves for HCC patients by the Barcelona Clinic Liver Cancer (BCLC) classification system (overall significance, $\mathrm{P}<0.001)$.

system had the highest value (c-index, 0.736 and 0.753 ). In patients treated with TATs $(\mathrm{n}=281)$, in 1-, 3- and 5-year c-index, PS-JIS system had the highest value (c-index, $0.842,0.843$ and 0.861 , respectively) (Table V).

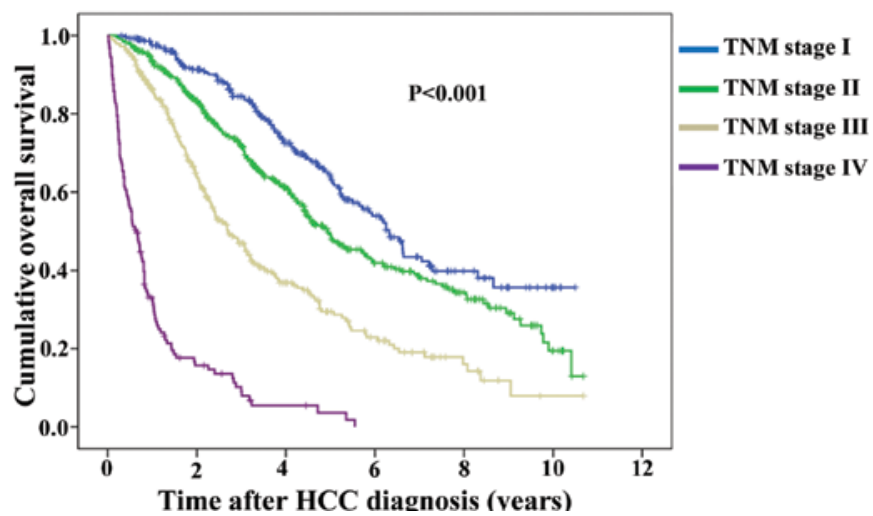

Figure 5. Kaplan-Meier survival curves for HCC patients by TNM classification system (overall significance, $\mathrm{P}<0.001$ ).

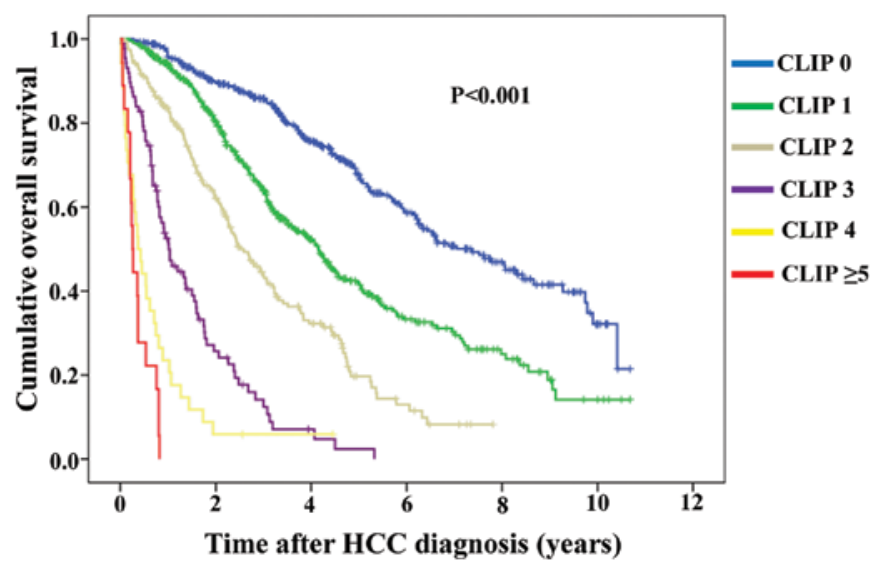

Figure 6. Kaplan-Meier survival curves for HCC patients by Cancer of the Liver Italian Program (CLIP) scoring system (overall significance, $\mathrm{P}<0.001$ ).

Subgroups analyses with regard to the effect of PS-JIS score stratified by JIS system. With the purpose of investigating the effect of PS-JIS, we performed subgroup analyses according to JIS system.

In patients with JIS 0 [ $\mathrm{n}=222$ : PS-JIS $0(\mathrm{n}=187)$, PS-JIS 1 $(\mathrm{n}=21)$ and PS-JIS $2(\mathrm{n}=14)$ ], JIS 1 [n=408: PS-JIS $1(\mathrm{n}=327)$, PS-JIS $2(\mathrm{n}=45)$ and PS-JIS $3(\mathrm{n}=36)$ ] and JIS 2 [n=297: PS-JIS 2 $(n=229)$, PS-JIS $3(n=39)$ and PS-JIS $4(n=29)]$, the differences in the three groups reached significance $(\mathrm{P}<0.001, \mathrm{P}<0.001$ and $\mathrm{P}=0.031$, respectively) (Fig. 7A-C). While in patients with JIS 3 [n=139: PS-JIS 3 (n=95), PS-JIS $4(n=24)$ and PS-JIS 5 $(\mathrm{n}=20)]$ and JIS 4 [n=86: PS-JIS $4(\mathrm{n}=45)$, PS-JIS $5(\mathrm{n}=13)$ and PS-JIS $6(n=28)]$, the differences in the three groups did not reach significance $(\mathrm{P}=0.301$ and $\mathrm{P}=0.343$, respectively). (Fig. 7D and E). Due to the small number of patients with JIS 5 $(n=18)$, we did not perform subgroup analysis in this group.

\section{Discussion}

The major difference among CLIP scoring system, BCLC classification system, TNM classification system and JIS system is that only BCLC classification included the ECOG-PS as a variable, although PS is a major prognostic factor for HCC patients (15). This factor is clinically impor- 
Table V. Comparison of discriminative ability using 1-, 3- and 5-year concordance index (c-index) among five prognostic systems.

\begin{tabular}{|c|c|c|c|c|c|c|}
\hline & \multicolumn{2}{|c|}{ 1-year } & \multicolumn{2}{|c|}{ 3-year } & \multicolumn{2}{|c|}{ 5-year } \\
\hline & c-index & $95 \% \mathrm{CI}$ & c-index & $95 \% \mathrm{CI}$ & c-index & $95 \% \mathrm{CI}$ \\
\hline \multicolumn{7}{|c|}{ All cases $(n=1170)$} \\
\hline JIS & 0.841 & $0.804-0.878$ & 0.797 & $0.768-0.826$ & 0.775 & $0.742-0.808$ \\
\hline PS-JIS & 0.847 & $0.814-0.880$ & 0.816 & $0.788-0.843$ & 0.808 & $0.778-0.838$ \\
\hline BCLC & 0.815 & $0.781-0.848$ & 0.778 & $0.748-0.807$ & 0.775 & $0.741-0.808$ \\
\hline TNM & 0.819 & $0.780-0.859$ & 0.754 & $0.723-0.786$ & 0.723 & $0.687-0.759$ \\
\hline CLIP & 0.817 & $0.775-0.859$ & 0.777 & $0.747-0.808$ & 0.776 & 0.743-0.809 \\
\hline \multicolumn{7}{|c|}{ SR $(n=205)$} \\
\hline JIS & 0.706 & $0.587-0.825$ & 0.717 & $0.631-0.803$ & 0.641 & $0.546-0.737$ \\
\hline PS-JIS & 0.718 & $0.603-0.832$ & 0.711 & $0.626-0.796$ & 0.661 & $0.567-0.755$ \\
\hline BCLC & 0.675 & $0.547-0.802$ & 0.657 & $0.566-0.748$ & 0.615 & $0.518-0.712$ \\
\hline TNM & 0.685 & $0.557-0.813$ & 0.674 & $0.583-0.765$ & 0.590 & $0.492-0.689$ \\
\hline CLIP & 0.739 & $0.618-0.859$ & 0.722 & $0.637-0.808$ & 0.681 & $0.589-0.773$ \\
\hline \multicolumn{7}{|c|}{ PATs $(n=632)$} \\
\hline JIS & 0.642 & $0.539-0.744$ & 0.700 & $0.651-0.749$ & 0.706 & $0.657-0.756$ \\
\hline PS-JIS & 0.714 & $0.623-0.805$ & 0.736 & $0.690-0.782$ & 0.753 & 0.706-0.799 \\
\hline BCLC & 0.740 & $0.647-0.834$ & 0.701 & $0.653-0.749$ & 0.716 & $0.667-0.765$ \\
\hline TNM & 0.621 & $0.517-0.725$ & 0.655 & $0.603-0.707$ & 0.646 & $0.593-0.700$ \\
\hline CLIP & 0.544 & $0.423-0.664$ & 0.662 & $0.610-0.713$ & 0.697 & $0.647-0.747$ \\
\hline \multicolumn{7}{|c|}{ TATs $(n=281)$} \\
\hline JIS & 0.834 & $0.784-0.883$ & 0.825 & $0.764-0.887$ & 0.827 & $0.737-0.917$ \\
\hline PS-JIS & 0.842 & 0.793-0.892 & 0.843 & $0.780-0.903$ & 0.861 & $0.785-0.938$ \\
\hline BCLC & 0.772 & $0.716-0.829$ & 0.809 & $0.741-0.878$ & 0.841 & $0.758-0.923$ \\
\hline TNM & 0.820 & $0.764-0.876$ & 0.775 & $0.706-0.843$ & 0.791 & $0.701-0.881$ \\
\hline CLIP & 0.838 & 0.786-0.889 & 0.820 & $0.760-0.880$ & 0.837 & $0.754-0.921$ \\
\hline
\end{tabular}

JIS, Japan Integrated Staging; PS-JIS, performance status combined Japan Integrated Staging; BCLC, Barcelona Clinic Liver Cancer; CLIP, Cancer of the Liver Italian Program; CI, confidence interval; SR, surgical resection; PATs, percutaneous ablative therapies; TATs, transcatheter arterial therapies.

tant for deciding treatment strategy for HCC and we believe that examining the effect of PS combined well known existing prognostic system on survival is worth reporting. Thus, we conducted the current analysis.

In our results, tumor number, Child-Pugh classification, PS, initial treatment modality, AST, ALP, AFP value and DCP value were significant predictors linked to OS in the multivariate analyses and c-index of PS-JIS was the highest at every time-point (1-, 3- and 5-year) for all cases. These results suggest that our proposed PS-JIS system can be a better prognostic system than the other existing prognostic systems. On the other hand, all P-values between adjacent groups in each system reached significance except for differences in PS-JIS score 4 and $5(\mathrm{P}=0.873)$, PS-JIS score 6 and $7(\mathrm{P}=0.199)$ and CLIP score 4 and 5 or $6(\mathrm{P}=0.082)$. This is probably due to the small sample sizes of these subgroups. Another possible reason is that PS-JIS (score range, 0-7) and CLIP score (score range, 0-6) are more complex scoring systems than the other prognostic systems.
In patients treated with SR, CLIP scoring system had the highest c-index among five prognostic systems at every timepoint in our analyses. On the other hand, Zhao et al (25) demonstrated that TNM staging system is a better staging model for $\mathrm{HCC}$ of Chinese population who received SR among seven currently applied staging systems including TNM, CLIP, BCLC, Okuda, CUPI, Tokyo score and CLIP score. As any staging system is constructed from selected prognostic factors of certain stage of HCC in a specific population, the predictive ability of the staging system could be considerably impaired if it is applied to another patient population $(7,26,27)$. The clinical outcome is closely associated with patient characteristics and subsequent therapeutic strategy $(7,26,27)$. As for etiology of liver disease, hepatitis $\mathrm{C}$ virus is in the majority in Japan, while hepatitis B virus is in the majority in China. In addition, treatment strategies for HCC are slightly different between Japan and China $(22,28)$. Discrepancies of our and their study results may be attributed to differences of the backgrounds between countries. 

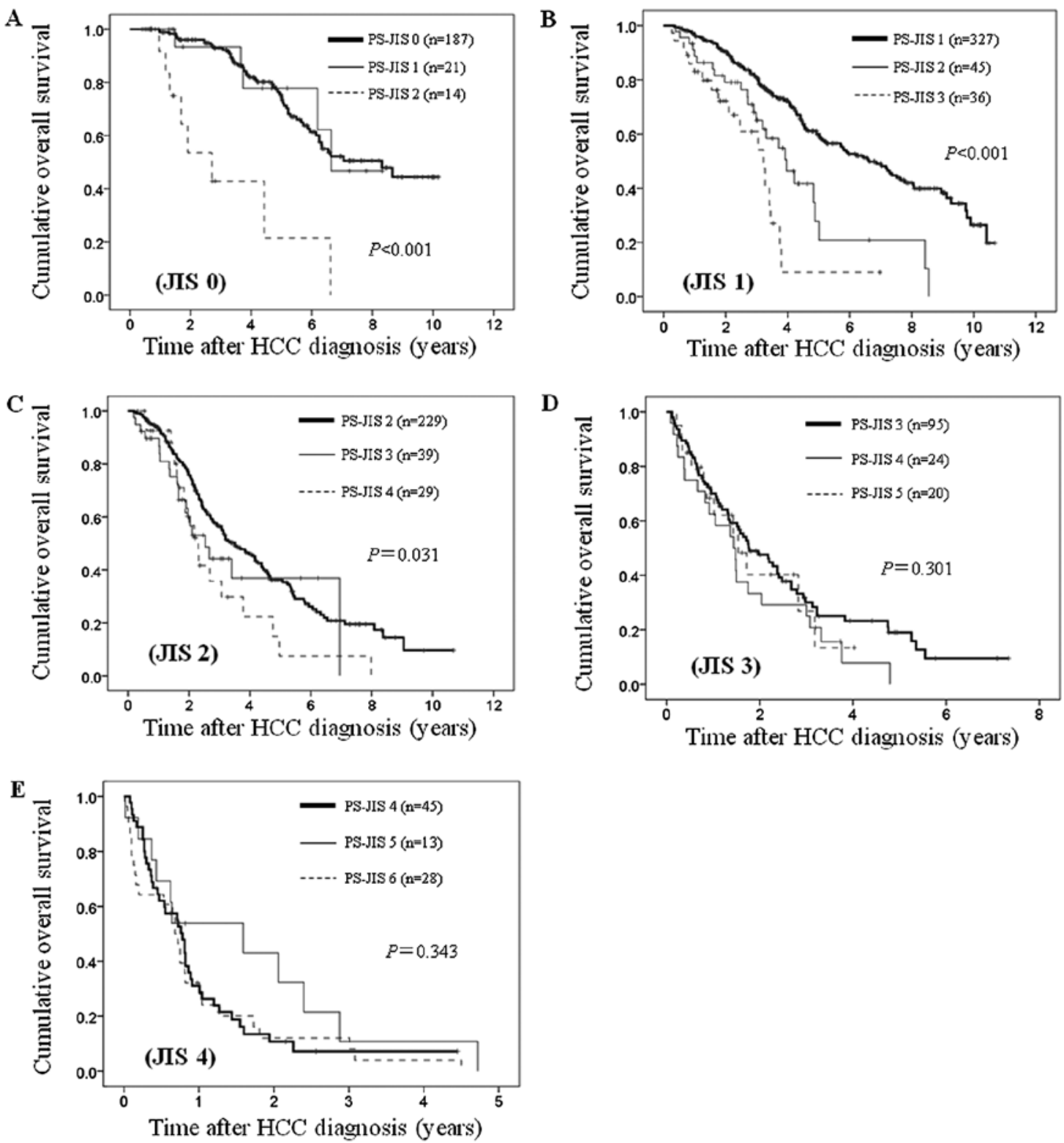

Figure 7. Kaplan-Meier survival curves in the three groups (groups of PS 0, PS 1 and PS >2) stratified by JIS score: (A) JIS 0, (B) JIS 1, (C) JIS 2, (D) JIS 3 and (E) JIS 4.

Furthermore, in our results, in patients treated with SR, only $6.3 \%$ (13/205) had poorer PS (PS >2) compared with the proportion of PS $>2$ of $11.7 \%$ (137/1170) for all cases. Thus, the effect of PS on survival may be diminished in this population as compared with other subgroups.

A previous study reported that the BCLC classification system shows a superior discriminatory power in their HCC patients who underwent RFA ( $n=112)$ among seven prognostic system, however, in the present study, in patients treated with PATs, in 1-year c-index, BCLC classification system had the highest value, while in 3- and 5-year c-index, PS-JIS system had the highest value (29). Likewise, Cho et al (18) demonstrated that CLIP system provided the best prognostic stratification for HCC patients who underwent transarterial chemoembolization $(\mathrm{n}=131)$, whereas in our analysis, in patients treated with TATs, in 1-, 3- and 5-year c-index, PS-JIS system had the highest value. As well as in patients treated with SR, these discrepancies can probably be explained in part by the difference of the baseline patient characteristics in the investigated populations.

According to sub-analyses stratified by JIS score, in early stages (JIS score 0,1 and 2), there was overall significance among three groups of PS 0,1 and $>2$ in terms of OS, whereas in advanced stages (JIS score 3 and 4), such significance was not found among three groups of PS 0,1 and $>2$. Our results indicate that especially in patients with early stage of HCC or less advanced LC, our proposed PS-JIS system can be a better prognostic system than the original JIS scoring system. In Japan, new emerging diagnostic imagings and the adequate selection of high-risk groups for HCC occurrence could enable detection of early stage HCC, potentially improving outcome (17). In that sense, our proposed PS-JIS system can be a promising scoring system.

We acknowledge several limitations in the current analyses. First, this is a single center retrospective study which included only Japanese HCC patients. Second, inter-observer 
bias for evaluating PS could exist although the PS scale was determined at the time of HCC diagnosis. Third, pathologic confirmation of HCC was not routinely performed except for cases who underwent SR. Caution should therefore be exercised in interpretation of our results, and our proposed staging system should be validated in another independent population. There are several missing values in the present study study. However, the number of patients with missing data was very small considering large sample of our study $(n=1,170)$, which may not effect on interpretation of our results.

In conclusion, our proposed PS-JIS score can be a useful prognostic system for HCC patients complicated with LC.

\section{Acknowledgements}

We would like to thank Haruko Takada for the data collection.

\section{References}

1. Poon D, Anderson BO, Chen LT, Tanaka K, Lau WY, Van Cutsem E, Singh H, Chow WC, Ooi LL, Chow P, et al; Asian Oncology Summit: Management of hepatocellular carcinoma in Asia: Consensus statement from the Asian Oncology Summit 2009. Lancet Oncol 10: 1111-1118, 2009.

2. No authors listed: A new prognostic system for hepatocellular carcinoma: a retrospective study of 435 patients: the Cancer of the Liver Italian Program (CLIP) investigators. Hepatology 28: 751-755, 1998.

3. Huitzil-Melendez FD, Capanu M, O'Reilly EM, Duffy A, Gansukh B, Saltz LL and Abou-Alfa GK: Advanced hepatocellular carcinoma: Which staging systems best predict prognosis? J Clin Oncol 28: 2889-2895, 2010.

4. Takanishi DM Jr, Severino R and Wong LL: The Cancer of the Liver Italian Program (CLIP) score: Validation of a new prognostic system for hepatocellular carcinoma. Hawaii Med J 66: 209-212, 2007.

5. Levy I and Sherman M; Liver Cancer Study Group of the University of Toronto: Staging of hepatocellular carcinoma: Assessment of the CLIP, Okuda, and Child-Pugh staging systems in a cohort of 257 patients in Toronto. Gut 50: 881-885, 2002

6. Llovet JM, Brú C and Bruix J: Prognosis of hepatocellular carcinoma: The BCLC staging classification. Semin Liver Dis 19: 329-338, 1999.

7. Marrero JA, Fontana RJ, Barrat A, Askari F, Conjeevaram HS, Su GL and Lok AS: Prognosis of hepatocellular carcinoma: Comparison of 7 staging systems in an American cohort. Hepatology 41: 707-716, 2005.

8. Cillo U, Vitale A, Grigoletto F, Farinati F, Brolese A, Zanus G, Neri D, Boccagni P, Srsen N, D'Amico F, et al: Prospective validation of the Barcelona Clinic Liver Cancer staging system. J Hepatol 44: 723-731, 2006.

9. Bruix J, Sherman M, Llovet JM, Beaugrand M, Lencioni R, Burroughs AK, Christensen E, Pagliaro L, Colombo M and Rodés J; EASL Panel of Experts on HCC; European Association for the Study of the Liver: Clinical management of hepatocellular carcinoma. Conclusions of the Barcelona-2000 EASL conference. J Hepatol 35: 421-430, 2001.

10. Kudo M, Chung H and Osaki Y: Prognostic staging system for hepatocellular carcinoma (CLIP score): Its value and limitations, and a proposal for a new staging system, the Japan Integrated Staging Score (JIS score). J Gastroenterol 38: 207-215, 2003.

11. No authors listed: Liver Cancer Study Group of Japan: The general rules for the clinical and pathological study of primary liver cancer. Jpn J Surg 19: 98-129, 1989.

12. Kudo M, Chung H, Haji S, Osaki Y, Oka H, Seki T, Kasugai H, Sasaki Y and Matsunaga T: Validation of a new prognostic staging system for hepatocellular carcinoma: The JIS score compared with the CLIP score. Hepatology 40: 1396-1405, 2004
13. Gomaa AI, Hashim MS and Waked I: Comparing staging systems for predicting prognosis and survival in patients with hepatocellular carcinoma in Egypt. PLoS One 9: e90929, 2014.

14. Bruix J and Sherman M; Practice Guidelines Committee, American Association for the Study of Liver Diseases: Management of hepatocellular carcinoma. Hepatology 42: 1208-1236, 2005.

15. Hsu CY, Lee YH, Hsia CY, Huang YH, Su CW, Lin HC, Lee RC, Chiou YY, Lee FY and Huo TI: Performance status in patients with hepatocellular carcinoma: Determinants, prognostic impact, and ability to improve the Barcelona Clinic Liver Cancer system. Hepatology 57: 112-119, 2013.

16. Montano-Loza AJ: Clinical relevance of sarcopenia in patients with cirrhosis. World J Gastroenterol 20: 8061-8071, 2014.

17. Osaki Y and Nishikawa H: Treatment for hepatocellular carcinoma in Japan over the last three decades: Our experience and published work review. Hepatol Res 45: 59-74, 2015.

18. Cho YK, Chung JW, Kim JK, Ahn YS, Kim MY, Park YO, Kim WT and Byun JH: Comparison of 7 staging systems for patients with hepatocellular carcinoma undergoing transarterial chemoembolization. Cancer 112: 352-361, 2008.

19. Kitai S, Kudo M, Minami Y, Ueshima K, Chung H, Hagiwara S, Inoue T, Ishikawa E, Takahashi S, Asakuma Y, et al: A new prognostic staging system for hepatocellular carcinoma: Value of the biomarker combined Japan integrated staging score. Intervirology 51 (Suppl 1): 86-94, 2008.

20. Yamasaki T, Kurokawa F, Shirahashi H, Kusano N, Hironaka K and Okita K: Percutaneous radiofrequency ablation therapy with combined angiography and computed tomography assistance for patients with hepatocellular carcinoma. Cancer 91: 1342-1348, 2001.

21. Park JW, Amarapurkar D, Chao Y, Chen PJ, Geschwind JF, Goh KL, Han KH, Kudo M, Lee HC, Lee RC, et al: Consensus recommendations and review by an International Expert Panel on Interventions in Hepatocellular Carcinoma (EPOIHCC). Liver Int 33: 327-337, 2013.

22. Kudo M, Izumi N, Kokudo N, Matsui O, Sakamoto M, Nakashima O, Kojiro M and Makuuchi M; HCC Expert Panel of Japan Society of Hepatology: Management of hepatocellular carcinoma in Japan: Consensus-Based Clinical Practice Guidelines proposed by the Japan Society of Hepatology (JSH) 2010 updated version. Dig Dis 29: 339-364, 2011.

23. Hanley JA and McNeil BJ: The meaning and use of the area under a receiver operating characteristic (ROC) curve. Radiology 143: 29-36, 1982.

24. Pencina MJ and D'Agostino RB: Overall $\mathrm{C}$ as a measure of discrimination in survival analysis: Model specific population value and confidence interval estimation. Stat Med 23: 2109-2123, 2004.

25. Zhao J, Yan T, Huang Z, Bi X, Zhao H, Zhou J, Li Z, Li Y, Li C and Cai J: Evaluations of seven different clinical staging systems for Chinese patients with hepatocellular carcinoma undergoing curative resection. Zhonghua Yi Xue Za Zhi 94: 903-907, 2014. (In Chinese).

26. Cillo U, Bassanello M, Vitale A, Grigoletto FA, Burra P, Fagiuoli S, D'Amico F, Ciarleglio FA, Boccagni P, Brolese A, et al: The critical issue of hepatocellular carcinoma prognostic classification: Which is the best tool available? J Hepatol 40: 124-131, 2004.

27. Collette S, Bonnetain F, Paoletti X, Doffoel M, Bouché O, Raoul JL, Rougier P, Masskouri F, Bedenne L and Barbare JC: Prognosis of advanced hepatocellular carcinoma: Comparison of three staging systems in two French clinical trials. Ann Oncol 19: 1117-1126, 2008

28. Omata M, Lesmana LA, Tateishi R, Chen PJ, Lin SM, Yoshida H, Kudo M, Lee JM, Choi BI, Poon RT, et al: Asian Pacific Association for the Study of the Liver consensus recommendations on hepatocellular carcinoma. Hepatol Int 4: 439-474, 2010.

29. Guglielmi A, Ruzzenente A, Pachera S, Valdegamberi A, Sandri M, D'Onofrio M and Iacono C: Comparison of seven staging systems in cirrhotic patients with hepatocellular carcinoma in a cohort of patients who underwent radiofrequency ablation with complete response. Am J Gastroenterol 103: 597-604, 2008. 